\title{
Peak Power Reduction of OFDM Signals with Sign Adjustment
}

\author{
Masoud Sharif, Vahid Tarokh, and Babak Hassibi
}

\begin{abstract}
It has recently been shown that significant reduction in the peak to mean envelope power (PMEPR) can be obtained by altering the sign of each subcarrier in a multicarrier system with $n$ subcarriers. However, finding the best sign not only requires a search over $2^{n}$ possible signs but also may lead to a substantial rate loss for small size constellations. In this paper, we first propose a greedy algorithm to choose the signs based on $p$-norm minimization and prove that the resulting PMEPR is guaranteed to be less than $c \log n$ where $c$ is a constant independent of $n$ for any $n$. This approach has lower complexity in each iteration compared to the derandomization approach of [1] while achieving similar PMEPR reduction. We further improve the performance of the proposed algorithm by enlarging the search space using pruning. Simulation results show that PMEPR of a multicarrier signal with 128 subcarriers can be reduced to within $1.6 \mathrm{~dB}$ of the PMEPR of a single carrier system.

In the second part of the paper, we address the rate loss by proposing a block coding scheme in which only one sign vector is chosen for $K$ different modulating vectors. The sign vector can be computed using the greedy algorithm in $n$ iterations. We show that the multi-symbol encoding approach can reduce the rate loss by a factor of $K$ while achieving the PMEPR of $c \log K n$, i.e., only logarithmic growth in $K$. Simulation results show that the rate loss can be made smaller than $\% 10$ at the cost of only $1 \mathrm{db}$ increase in the resulting PMEPR for a system with 128 subcarriers.
\end{abstract}

Index Terms-Orthogonal frequency division multiplexing (OFDM), peak to average power reduction, greedy algorithms, coding.

\section{INTRODUCTION}

$\mathbf{M}$ ULTICARRIER modulation has been proposed for high speed wireless and wireline communications in several standards such as IEEE 802.11(a),(g), WiMax, xDSL, and Digital Video/Audio Broadcasting. The main advantage of this modulation over single carrier systems is the simplicity of channel equalization for frequency selective channels. However, the main drawback of multicarrier systems is their high

Paper approved by N. Al-Dhahir, the Editor for Space-Time, OFDM and Equalization of the IEEE Communications Society. Manuscript received January 6, 2008; revised April 27, 2008.

M. Sharif is with the Department of Electrical and Computer Engineering, Boston University, MA (e-mail: sharif@bu.edu; masoud@systems.caltech.edu).

V. Tarokh is with the Department of Electrical Engineering, Harvard University, Cambridge, MA (e-mail: vahid@seas.harvard.edu).

B. Hassibi is with the department of Electrical Engineering, California Institute of Technology, Pasadena, CA (e-mail: hassibi@systems.caltech.edu).

The work of M. Sharif was supported by the National Science Foundation under grant no. CCF-0728484. The work of V. Tarokh was also supported by the National Science Foundation under grant no. CCF-0728572. The work of B. Hassibi has been supported by the National Science Foundation through grants CCR-0133818 and CCR-0326554, by the Office of Naval Research under grant ONR N00014-08-1-0747, by the David and Lucille Packard Foundation, and by Caltech's Lee Center for Networking.

Digital Object Identifier 10.1109/TCOMM.2009.07.080012 peak to mean envelope power ratio (PMEPR) as $n$ subcarriers may add up constructively and produce large peaks of order $n$. In practice $n$ is large (e.g., of the order of hundred) and therefore, the power amplifier should be highly linear which significantly hampers its power efficiency and significantly reduces the battery life time [2], [3].

Several methods have been developed for PMEPR reduction such as coding, deliberate clipping, selective mapping (SLM), reserved carriers, and tone injection (see [4] and references there in). The reduction in PMEPR, of course, comes at a price in terms of coding rate, average power, signal distortion, and/or bandwidth [5]-[13]. In this paper, we propose a coding scheme that reduces the PMEPR at the expense of a rate hit.

It has been recently shown that altering the sign of each subcarrier for any given data vector can significantly reduce the PMEPR [1]. In particular, for large $n$ and when each subcarrier is modulated by a symmetric $q$-ary constellation ${ }^{1}$, the PMEPR can be reduced to a constant independent of $n$ at the price of a rate loss equal to $\log _{q} 2^{2}$. This result further motivates ( $i$ ) developing algorithms to find a good choice of the sign vector efficiently as the search for the optimum sign requires a search over $2^{n-1}$ choices, and (ii) proposing methods to reduce the rate loss without too much sacrificing the PMEPR.

Efficient search algorithms for the signs with polynomial complexity have been proposed. In particular, a derandomization method has been proposed to find the signs iteratively and can guarantee a PMEPR of $c \log n$ for any $n$ where $c \geq 1$ is a constant independent of $n$ [1], [14]. For large $n$, Litsyn and Shpunt [15] also proposed a novel technique that searches exhaustively over a much smaller set of codewords, i.e., a set of size $n^{\log n}$ rather the set of $2^{n}$ possible sign vectors, with PMEPR guarantee of $\log n$. The rate loss can be also addressed by using a fraction of the $n$ possible signs [16], by searching over a small set of sign vectors as in [15], or use a fix set of predefined sign vectors such as Hadamard matrices as in SLM [17]-[20]. Other constellation shaping algorithms have been also proposed based on Trellis shaping [21].

In this paper we propose an iterative greedy algorithm based on $p$-norm minimization that has much less complexity at each iteration compared to that of derandomization method and can provide the same worst case guarantee on the PMEPR. As the complexity of each iteration is negligible, we can further improve the performance by enlarging the search space

\footnotetext{
${ }^{1} \mathrm{~A}$ symmetric constellation is defined as a constellation that includes both points $A$ and $-A$.

${ }^{2}$ Rate is defined as $1 / n$ of the logarithm of the cardinality of the set of all codewords as defined in Section 2, Eq. (4). Here rate of 1 refers to the full rate code.
} 
considered by the greedy algorithm. This can significantly improve the performance of the greedy algorithm and reduce the PMEPR within $2 \mathrm{~dB}$ of the PMEPR of single carrier systems.

In the second part of the paper, we address the rate loss associated with not sending information over $n$ signs of the subcarriers. We use a multi-symbol encoding approach and choose only one sign vector to reduce the PMEPR of $K$ different modulating codewords simultaneously. Using the greedy algorithm with $n$ iterations, we show that our approach reduces the rate loss by a factor of $K$ while not sacrificing PMEPR too much. In particular, we show that the resulting PMEPR is bounded by $c \log K n$ for any $n$ and $K$ with a rate loss of only $\frac{1}{K} \log _{q} 2$ for a $q$-ary constellation. For large $n$, we can therefore construct codes of rate $R=1-O\left(\frac{1}{n^{\gamma}}\right)$ and PMEPR of less than $c(\gamma+1) \log n$ for any constant $\gamma \geq 1$.

The paper is organized as follows: Section 2 introduces our notation and states the PMEPR minimization problem. Section 3 deals with the greedy algorithm and its variations to choose $n$ signs such that the PMEPR of a codeword is minimized. Section 4 introduces the multi-symbol encoding method to improve the rate loss by using $n$ signs for more than one codewords. Finally Section 5 concludes the paper.

\section{Definitions And Problem Statement}

The complex envelope of a multicarrier signal with $n$ subcarriers may be represented as,

$$
s_{C}(\theta)=\sum_{i=1}^{n} c_{i} e^{j \theta i}, \quad 0 \leq \theta<2 \pi,
$$

where $C=\left(c_{1}, \ldots, c_{n}\right)$ is the complex modulating OFDM symbol (or a codeword) with entries from a given complex $q$-ary constellation. The admissible modulating vectors are called codewords and the ensemble of all possible codewords constitute the code $\mathcal{C}$. Then, the PMEPR of each codeword $C$ in the code family $\mathcal{C}$ may be defined as,

$$
\operatorname{PMEPR}_{\mathcal{C}}(C)=\max _{0 \leq \theta<2 \pi} \frac{\left|s_{C}(\theta)\right|^{2}}{E\left\{\|C\|_{2}^{2}\right\}} .
$$

Similarly, $\mathrm{PMEPR}_{\mathcal{C}}$ is defined as the maximum of Eq. (2) over all codewords in $\mathcal{C}$. If $c_{i}$ 's are chosen independently and identically from some constellation with average power $E_{a v}$ then $E\left\{\|C\|_{2}^{2}\right\}=n E_{a v}$.

Even though the worst case PMEPR is of the order $n$ when $c_{i}$ 's are chosen from a constellation such as QAM, it is shown that with high probability the PMEPR of a random codeword is $\log n$ almost surely [22]-[25]. This implies that the PMEPR is not as bad as what is predicted by the worst case and its distribution should be taken into consideration. Fig. 1 compares the complementary cumulative distribution function (CCDF) of PMEPR for a multicarrier system with $n=128$ and using 64QAM constellation with that of a single carrier system. As shown in Fig. 1, with probability $1-10^{-3}$, the PMEPR of the multicarrier system is less than or equal to 12.5 as opposed to 2.3 for the single carrier system ${ }^{3}$.

\footnotetext{
${ }^{3}$ It is worth mentioning that the PMEPR of the OFDM signal is obtained using oversampling of the signal and the PMEPR of the single carrier system is calculated using Nyquist rate sampling and assuming that a rectangular time-domain windowing has been applied to the signal.
}

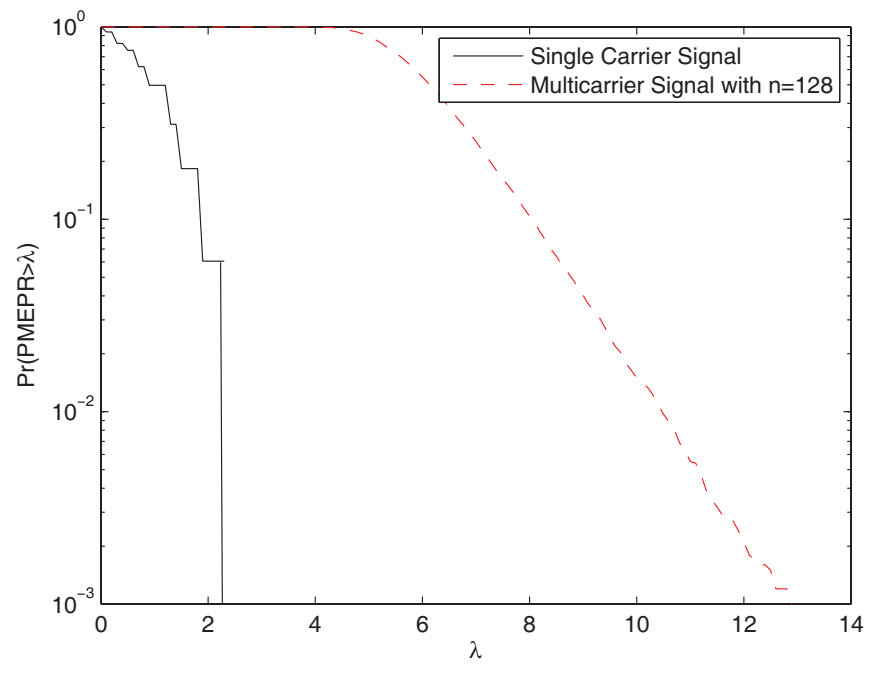

Fig. 1. Comparison of $\operatorname{Pr}(\mathrm{PMEPR}>\lambda)$ for a multicarrier system with $n=128$ and a single carrier system using 64QAM constellation for 5000 random codewords.

In [1], it has been shown that adjusting the sign of each subcarrier is a promising technique for PMEPR reduction of multicarrier signals and leads to the proof for the existence of nonvanishing to zero rate codes with PMEPR bounded by a constant (see also [25]). The main idea is to choose a sign $\epsilon_{i}$ for each subcarrier to minimize the maximum absolute value of the signal. Hence, given the codeword $C=\left(c_{1}, \ldots, c_{n}\right)$, the following problem should be solved,

$$
\min _{\epsilon} \max _{0 \leq \theta \leq 2 \pi}\left|\sum_{i=1}^{n} \epsilon_{i} c_{i} e^{j \theta i}\right|
$$

where $\epsilon=\left(\epsilon_{1}, \ldots, \epsilon_{n}\right)$ and $\epsilon_{i} \in\{+1,-1\}$.

It is worth mentioning that these signs do not carry any information and therefore the receiver simply ignores the sign of each subcarrier. This implies that by using the sign of each subcarrier to reduce the PMEPR, we can construct a code with $(q / 2)^{n}$ codewords (out of $q^{n}$ ) that has a very small PMEPR [1]. To quantify this reduction in the number of codewords, we may define the rate of a code $\mathcal{C}$ as

$$
R=\frac{1}{n} \log _{q}|\mathcal{C}|,
$$

where $c_{i}$ 's are chosen from a $q$-ary constellation and $|\mathcal{C}|$ denotes the cardinality of the set $\mathcal{C}$. Clearly based on our definition of rate, full rate codes refer to $R=1$ for any $q$ and if $n$ signs are used for PMEPR reduction, the resulting rate would be $1-\log _{q} 2$.

Of course finding the optimum solution for the combinatorial optimization problem in (3) has exponential complexity. In [1], [16], an algorithm is proposed to find the signs with linear complexity which guarantees that the PMEPR is less than $c \log n$. Recently, in [15], it is shown that by searching over a small subset of $2^{n}$ signs, PMEPR of order $\log n$ can be achieved with $n^{\log n}$ complexity. The main goal of this paper is to investigate polynomial time algorithms to choose the signs and further reduce the gap between the PMEPR of multicarrier and single carrier signals while not reducing the rate of the system significantly. 


\section{A Greedy Algorithm to Choose the Signs}

In this section, we propose a greedy algorithm to choose the signs that guarantees the PMEPR of $c \log n$. This is done iteratively by using a $p$-norm minimization as opposed to minimizing the conditional probability for the derandomization method as in [1]. We then propose two variations of our algorithm to further improve the reduction of PMEPR by increasing the computational complexity of the algorithm. These methods are based on enlarging the search and pruning the tree of $2^{n}$ signs using the $p$-norm metric.

Following [1], we first change the problem in (3) and instead of looking at the maximum of $s_{C}(\theta)$ over $0 \leq \theta \leq 2 \pi$, we look at its maximum over uniform samples of $\theta$ at $\theta_{u}=\frac{2 \pi u}{k n}$ for $u=1, \ldots, k n$ where $k>1$ is the oversampling factor ${ }^{4}$. Therefore, the problem can be stated as:

$$
\min _{\epsilon} \max _{1 \leq u \leq 2 k n}\left|\sum_{i=1}^{n} \epsilon_{i} a_{u i}\right|
$$

where $a_{u i}$ is defined as,

$$
a_{u i}= \begin{cases}\operatorname{Re}\left\{c_{i} e^{j \theta_{u} i}\right\} & 1 \leq u \leq k n, \\ \operatorname{Im}\left\{c_{i} e^{j \theta_{u} i}\right\} & k n+1 \leq u \leq 2 k n\end{cases}
$$

In other words, we would like to solve

$$
\min _{\epsilon_{i}^{2}=1, i=1, \ldots, n}\|A \epsilon\|_{\infty}=\min _{\epsilon_{i}^{2}=1, i=1, \ldots, n}\left\|\sum_{i=1}^{n} \mathbf{a}_{i} \epsilon_{i}\right\|_{\infty}
$$

where $A^{t}=\left[\mathbf{a}_{i}\right]$ is a $2 k n \times n$ real matrix and $\mathbf{a}_{i}=\left[a_{u i}\right]$. Without loss of generality we assume that $\left|a_{u i}\right|<1$ (which can be done by scaling the constellation).

It is known that for large $n$ and for any codeword $C$, there exists a choice of sign vector $\epsilon$ such that the PMEPR is bounded by a constant independent of $n$. Moreover, randomly choosing signs will lead to a PMEPR of $\log n$ with high probability. The challenge however is to find a sign vector efficiently that "guarantees" the PMEPR to be either bounded by $\log n$ or constant.

In [1], a deterministic algorithm is proposed to design the signs using derandomization (see also [26]). The algorithm chooses the signs recursively based on the knowledge of all $\mathbf{a}_{i}$ 's. In fact, at $s$ 'th step, we choose the sign that minimizes the conditional probability that $\|A \epsilon\|_{\infty}$ is greater than some threshold $\lambda$ and given $\epsilon_{1}, \ldots, \epsilon_{s-1}$. Since finding the conditional probability can be quite involved, we can use the Chernoff bound instead. This leads to the following algorithm (see [1] for details).

Derandomization Algorithm [1]. For any codeword $C=$ $\left(c_{1}, \ldots, c_{n}\right)$, let $a_{u i}$ be as in (6). Then $\epsilon_{1}=1$, and $\epsilon_{s}$ 's are recursively determined as

$$
\begin{array}{r}
\epsilon_{s}=-\operatorname{sign}\left\{\sum_{u=1}^{2 k n} \sinh \left\{\alpha^{*} \sum_{r=1}^{s-1} \epsilon_{r} a_{u r}\right\} \sinh \left(\alpha^{*} a_{u s}\right)\right. \\
\left.\prod_{r=s+1}^{n} \cosh \left(\alpha^{*} a_{u r}\right)\right\} .
\end{array}
$$

for $s=2, \ldots, n$, where $\alpha^{*}=\sqrt{\frac{2 \log 4 k n}{n}}$.

\footnotetext{
${ }^{4}$ We choose $k=4$ in our simulations.
}

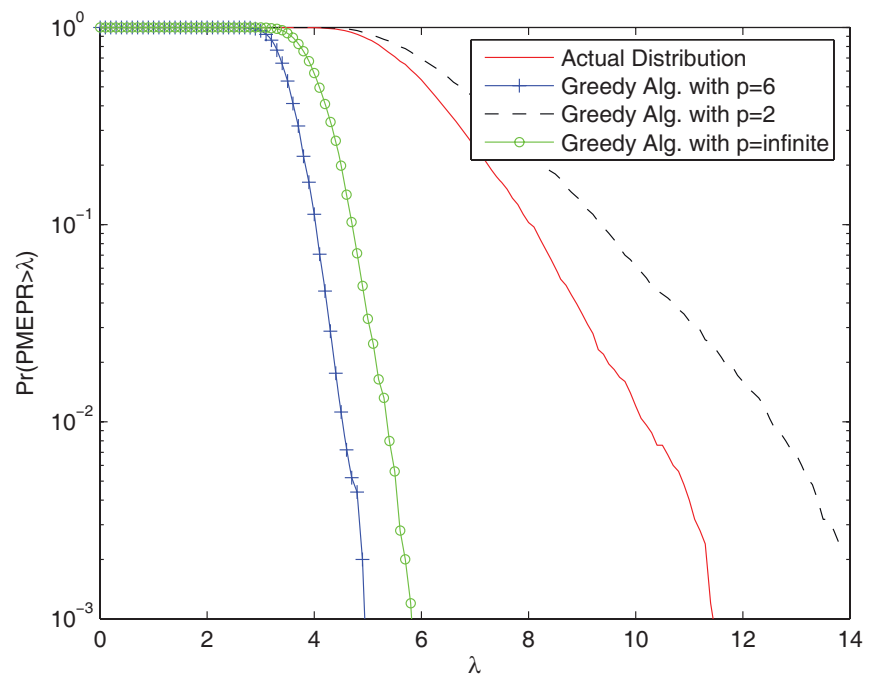

Fig. 2. Comparison of $\operatorname{Pr}(\mathrm{PMEPR}>\lambda)$ for $n=128$ and using Greedy algorithm for different value of $p$ and for 5000 codewords.

It is shown that the PMEPR of the resulting codeword $\left(\epsilon_{1} c_{1}, \ldots, \epsilon_{n} c_{n}\right)$ is less than $c \log n$ for any $n$ where $c$ only depends on the constellation. It is worth mentioning by following the approach in [15], we could improve the factor $c$ in the upper bound using more than two axes (i.e., real and imaginary).

The only drawback of the derandomization Algorithm is that the computation at each step involves taking cosine hyperbolic $k n$ times which can be prohibitive for large number of subcarriers. In order to simplify the computation at each step, one may try choosing the signs in a greedy manner in which at each step the sign that minimizes $\left\|\sum_{i=1}^{s} \mathbf{a}_{i} \epsilon_{i}\right\|_{\infty}$ is chosen given $\epsilon_{1}, \ldots, \epsilon_{s-1}$. Interestingly, we can improve the performance by changing the infinity norm to norm $p$. Fig. 2 shows the performance of this method using different norms. It is clear that for $n=128$, using $p=6$ leads to quite a large improvement.

We can in fact justify this behavior analytically. The main result of this section is to obtain a bound on the PMEPR obtained from greedily minimizing the metric $\left\|\sum_{i=1}^{s} \epsilon_{i} \mathbf{a}_{i}\right\|_{p}^{p}$. In particular, we show that the optimal $p$ is $\log 2 k n$, which yields a PMEPR of $c \log n$ for any $n$. Here is the algorithm:

Greedy Algorithm: Let $\epsilon_{1}=1$, and having chosen $\epsilon_{2}, \ldots, \epsilon_{s-1}$, then

$$
\epsilon_{s}=\arg \min _{\epsilon_{s} \in\{+1,-1\}}\left\|\sum_{i=1}^{s} \mathbf{a}_{i} \epsilon_{i}\right\|_{p}^{p},
$$

The next Theorem provides a worst case guarantee on the performance of the greedy algorithm and provides insight into how to choose the $p$ leading to the best performance.

Theorem 1: For any $p$ greater than 2, and assuming all the entries of $A_{2 k n \times n}=\left[a_{i, j}\right]$ are $\left|a_{i, j}\right| \leq 1$, the choice of $\epsilon=\left(\epsilon_{1}, \ldots, \epsilon_{n}\right)$ given by Greedy algorithm ensures that

$$
\|A \epsilon\|_{\infty} \leq(2 k n)^{1 / p} \sqrt{p n}
$$

for any $n$. If $p=\log 2 k n$, then the upper bound is $e \sqrt{n \log k n}$.

Proof: We present the proof when $p$ is even for simplicity. If $p$ is odd, we can follow a similar approach. Assume 
$\epsilon_{1}, \ldots, \epsilon_{r-1}$ have already been determined. We define the sequence $B_{r-1}^{p}=\frac{1}{2 k n}\left\|\sum_{i=1}^{r-1} \mathbf{a}_{i} \epsilon_{i}\right\|_{p}^{p}$. Using (8), we now find a bound on $B_{r}$ based on $B_{r-1}$.

Denoting $\sum_{i=1}^{r-1} \mathbf{a}_{i} \epsilon_{i}=\left(y_{1}, \ldots, y_{2 k n}\right)^{t}$ and $\mathbf{a}_{r}=$ $\left(a_{1, r}, \ldots, a_{2 k n, r}\right)^{t}$, we may write,

$$
\begin{aligned}
2 k n B_{r}^{p} & =\min \left\{\sum_{j=1}^{2 k n}\left(y_{j}-a_{j, r}\right)^{p}, \sum_{j=1}^{2 k n}\left(y_{j}+a_{j, r}\right)^{p}\right\} \\
& \leq \frac{1}{2}\left(\sum_{j=1}^{2 k n}\left(y_{j}+a_{j, r}\right)^{p}+\left(y_{j}-a_{j, r}\right)^{p}\right) \\
& \leq \frac{1}{2}\left(\sum_{j=1}^{2 k n}\left(y_{j}+1\right)^{p}+\left(y_{j}-1\right)^{p}\right)
\end{aligned}
$$

The last equality follows from the fact that $\left|a_{i, r}\right| \leq 1$ and also using the inequality

$$
\left(y_{j}+a_{j, r}\right)^{p}+\left(y_{j}-a_{j, r}\right)^{p} \leq\left(y_{j}+1\right)^{p}+\left(y_{j}-1\right)^{p}
$$

for $p \geq 2$ and $\left|a_{r, j}\right| \leq 1$. The bound can be proved using the convexity of the left hand side of (11) and therefore its maximum is attained on the boundary. We can further bound (10) as follows,

$$
\begin{aligned}
B_{r}^{p} & \leq \frac{1}{2} \frac{1}{2 k n} \sum_{j=1}^{2 k n}\left(y_{j}+1\right)^{p}+\left(y_{j}-1\right)^{p} \\
& =\frac{1}{2} \frac{1}{2 k n} \sum_{j=1}^{2 k n} \sum_{r=0}^{p}\left(\begin{array}{l}
s \\
r
\end{array}\right) y_{j}^{r}\left(1+(-1)^{p-r}\right) \\
& \leq \frac{1}{2} \sum_{r=0}^{p}\left(\begin{array}{l}
s \\
r
\end{array}\right)\left(\frac{1}{2 k n} \sum_{j=1}^{2 k n} y_{j}^{p}\right)^{r / p}\left(1+(-1)^{p-r}\right) .
\end{aligned}
$$

where (12) is obtained using the fact that

$$
\left(\frac{1}{M} \sum_{i=1}^{M} x_{i}^{2}\right)^{\frac{1}{2}} \leq\left(\frac{1}{M} \sum_{i=1}^{M} x_{i}^{p}\right)^{\frac{1}{p}}
$$

for $p \geq 2$. Therefore,

$$
\begin{aligned}
B_{r}^{p} \leq & \frac{1}{2}\left(\left(\frac{1}{2 k n} \sum_{j=1}^{2 k n} y_{j}^{p}\right)^{1 / p}+1\right)^{p} \\
& +\frac{1}{2}\left(\left(\frac{1}{2 k n} \sum_{j=1}^{2 k n} y_{j}^{p}\right)^{1 / p}-1\right)^{p} \\
\leq & \frac{1}{2}\left\{\left(B_{r-1}+1\right)^{p}+\left(B_{r-1}-1\right)^{p}\right\} \\
= & \frac{1}{2} \sum_{j=0}^{p / 2}\left(\begin{array}{c}
p \\
2 j
\end{array}\right) B_{r-1}^{2 j} \\
\leq & \left(B_{r-1}^{2}+p\right)^{p / 2}
\end{aligned}
$$

where (14) follows by expanding the right hand side of (13) and using the fact that

$$
\frac{1}{2}\left(\begin{array}{c}
p \\
2 j
\end{array}\right) \leq\left(\begin{array}{c}
p / 2 \\
p / 2-j
\end{array}\right) \times p^{p / 2-j}=\left(\begin{array}{c}
p / 2 \\
j
\end{array}\right) \times p^{p / 2-j} .
$$

We can therefore obtain the following recursive bound on $B_{r}: B_{r}^{2} \leq B_{r-1}^{2}+p$. Noting that $B_{1} \leq 1$, we conclude that $B_{n} \leq \sqrt{n p}$, and therefore,

$$
\begin{aligned}
\left\|\sum_{i=1}^{n} \mathbf{a}_{i} \epsilon_{i}\right\|_{\infty} & \leq\left\|\sum_{i=1}^{n} \mathbf{a}_{i} \epsilon_{i}\right\|_{p} \\
& =(2 k n)^{1 / p} B_{n} \\
& =(2 k n)^{1 / p} \sqrt{p n} .
\end{aligned}
$$

Finally, letting $p=\log 2 k n$ in (15) completes the proof of the theorem.

We would like to comment that the factor $e$ in the upper bound of Theorem 1 can be improved to one using the approach of [15].

Theorem 1 implies that for a given codeword $\left(c_{1}, \ldots, c_{n}\right)$, if we choose a sign vector using Greedy algorithm with $p=$ $\log 2 k n$, we can guarantee that,

$$
\max _{1 \leq u \leq k n}\left|\sum_{i=1}^{n} \epsilon_{i} c_{i} e^{j 2 \pi i \theta_{u}}\right| \leq \max _{1 \leq i \leq n}\left|c_{i}\right| e \sqrt{n \log k n},
$$

where $\theta_{u}=\frac{2 \pi u}{k n}$. As shown in [27]-[29] (see also Chapter 4 of [4]), we can bound the maximum of $s_{C}(\theta)$ over $0 \leq$ $\theta \leq 2 \pi$, by its $2 k n$ uniform samples at $\theta_{u}$ 's. We can therefore conclude that the PMEPR of the resulting codeword $C_{\epsilon}=$ $\left(\epsilon_{1} c_{1}, \epsilon_{2} c_{2}, \ldots, \epsilon_{n} c_{n}\right)$ is bounded by,

$$
\operatorname{PMEPR}\left(C_{\epsilon}\right) \leq \frac{e^{2} P_{\text {max }}}{P_{a v} \cos ^{2} \frac{\pi}{2 k}} \log 2 k n,
$$

for any $n$ where $P_{\max }=\max _{1 \leq i \leq n}\left|c_{i}\right|^{2}$. The next corollary states the result.

Corollary 1. Given that $c_{i}$ 's are chosen from a constellation of size $q$, maximum energy $P_{\max }$, and average energy $P_{a v}$, we can construct a code using the Greedy algorithm with $p=$ $\log 2 k n$ that has a rate $R=1-\log _{q} 2$ and guarantees $a$ PMEPR of $c \log 2 k n$ for any $n$ where $c$ is equal to $\frac{e^{2} P_{\max }}{P_{a v} \cos ^{2} \frac{\pi}{2 k}}$ and is independent of $n$.

Fig. 3 compares the performance of greedy algorithm and derandomization method. Clearly, there is still a gap between the PMEPR of the multicarrier system (i.e., 4.8) and that of the single carrier systems (i.e., 2.3). More precisely, we would like to see whether we can efficiently find a better choice of the signs that further reduces the PMEPR and approaches the CCDF of the single carrier system. Since the greedy algorithm can be implemented very efficiently, we could increase the size of the search space as considered in the next subsection.

\section{A. Improving the Search by Pruning}

Motivated by the low complexity of the greedy algorithm, we can enlarge the search space to find a better choice for the signs while slightly increasing the complexity. Here, we consider two variations/improvements of the greedy algorithm.

Pruning Algorithm 1: In the first approach, we search over all the possible signs for the first $m$ subcarriers, i.e., $\epsilon_{1}, \ldots, \epsilon_{m}$, and then we use the greedy Algorithm to find the choice of the signs for the remaining $n-m$ signs. Finally we choose the sign vector (out of $2^{m-1}$ possible choices as 


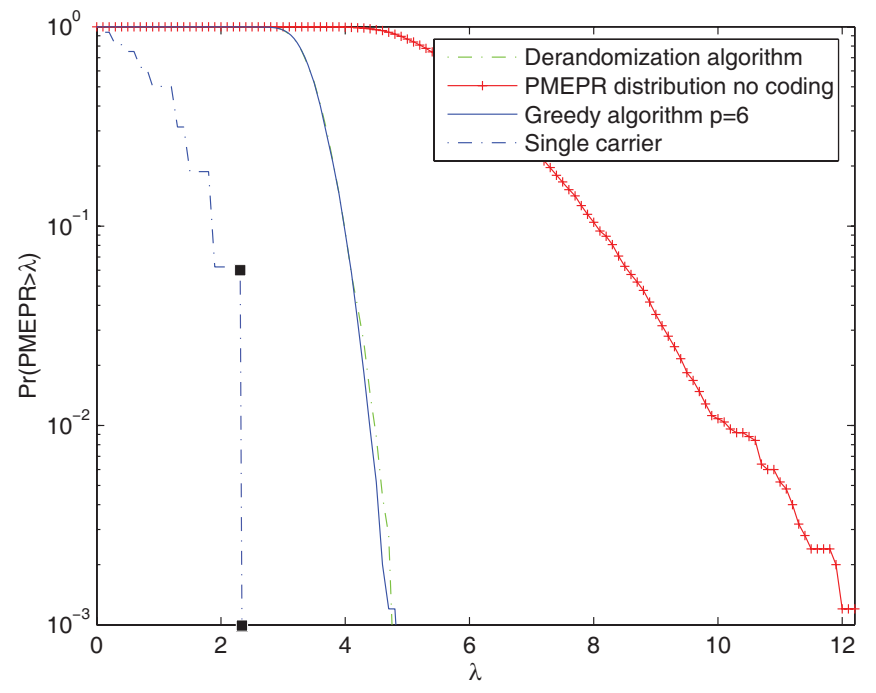

Fig. 3. A comparison of the PMEPR reduction using Algorithm 1, 2, and 3 for 5000 random codewords and for 64QAM constellation.

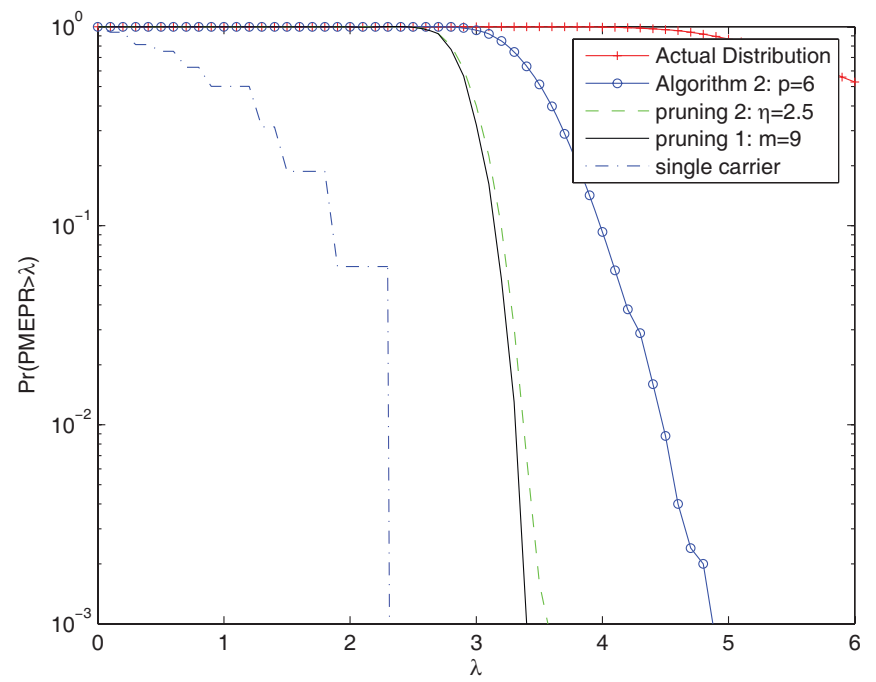

Fig. 4. Comparison of $\operatorname{Pr}(\mathrm{PMEPR}>\lambda)$ for $n=128$ using the pruning algorithms compared to the greedy algorithm without pruning with $p=6$ for 5000 random codewords.

$\epsilon_{1}=1$ ) that has the least PMEPR. This of course increases the complexity of the greedy algorithm by a factor of $2^{m}$ as it runs the greedy algorithm for the first $2^{m}$ choices of the signs. Fig. 4 shows the performance of this scheme for different $m$ 's. It can be seen that the PMEPR has been reduced from 4.8 to 3.4 at the cost of additional computational complexity at the transmitter.

Pruning Algorithm 2: In the second approach, we consider the metric at the $s$ 'th stage to be $\left\|\sum_{i=1}^{s} \mathbf{a}_{i} \epsilon_{i}\right\|_{p}^{p}$. Instead of just looking at the choice of the sign that minimizes the metric at each stage, we keep the sign choices as long as the metric is less than some threshold value. One legitimate choice of the threshold would be the value of the metric by running Algorithm 2. In order to allow for more sign vectors, we may increase the threshold at each stage by some value (say $\eta$ ). At the end of the algorithm, we choose the best sign vector in terms of PMEPR. Fig. 4 shows the resulting PMEPR improvement for different values of $\eta$. Clearly, the PMEPR is improved from 12.5 to 3.4 for the multicarrier system with 128 subcarriers and its PMEPR is just $1.6 \mathrm{~dB}=10 \log (3.4 / 2.3)$ worse than the single carrier system. This motivates further investigation for more effective algorithms to choose the sign vector with less complexity. Moreover, the question of how much further we can improve the PMEPR remains open.

\section{Improving The Coding RATE: Multi-Symbol ENCODING}

The main disadvantage in the proposed coding scheme based on sign adjustment is the rate loss specially for small size constellations. For example, if $n$ signs are used for a QPSK constellation, the significant PMEPR reduction is obtained at the price of $\% 50$ rate loss. In [16], using only a fraction of $n$ signs, say $\frac{n}{r}$, is used for PMEPR of reduction of a codeword. While this approach is reducing the rate loss by a factor of $r$, i.e., $R=1-\frac{1}{r} \log _{q} 2$, it can only a guarantee a PMEPR of $c r \log n$, i.e., the worst case is increased by a factor of $r$.

In this section, we propose a scheme that can improve the rate loss without too much sacrificing the PMEPR. The main idea is to use $n$ signs (or even less) for simultaneously reducing the PMEPR of $K>1$ codewords. This of course increases the complexity, however thanks to the simplicity of the iterative greedy algorithm, we show that a choice of sign vector can be computed efficiently with only $n$ iterations and can guarantee a PMEPR of $c \log n K$. The method clearly increases the coding rate to $1-\frac{1}{K} \log _{q} 2$ while slightly sacrificing the PMEPR gain, i.e., from $c \log n$ to $c \log n K$ implying only a logarithmic growth in $K$.

The problem can be stated as follows: Given the codewords $C^{k}=\left(c_{1}^{k}, \ldots, c_{n}^{k}\right)$ for $k=1, \ldots, K$ where $c_{i}$ 's are chosen from a $q$-ary constellation, we are interested in the solution of the following polynomially constrained optimization problem,

$$
\begin{array}{r}
\min _{\epsilon}\left\{\max _{0 \leq \theta \leq 2 \pi}\left|\sum_{i=1}^{n} \epsilon_{i} c_{i}^{1} e^{j \theta i}\right|, \max _{0 \leq \theta \leq 2 \pi}\left|\sum_{i=1}^{n} \epsilon_{i} c_{i}^{2} e^{j \theta i}\right|, \ldots,\right. \\
\left.\max _{0 \leq \theta \leq 2 \pi}\left|\sum_{i=1}^{n} \epsilon_{i} c_{i}^{K} e^{j \theta i}\right|\right\}
\end{array}
$$

where $\epsilon=\left(\epsilon_{1}, \ldots, \epsilon_{n}\right)$ and $\epsilon_{i} \in\{+1,-1\}$.

Following the derivation of (5), our problem can be stated as:

$$
\min _{\epsilon} \max _{\substack{1 \leq u \leq 2 k n \\ 1 \leq r \leq K}}\left|\sum_{i=1}^{n} \epsilon_{i} a_{u i}^{r}\right|
$$

where $a_{u i}^{r}$ is defined as,

$$
a_{u i}^{r}= \begin{cases}\operatorname{Re}\left\{c_{i}^{r} e^{j \theta_{u} i}\right\} & 1+(r-1) 2 k n \leq u \leq(2 r-1) k n, \\ \operatorname{Im}\left\{c_{i}^{r} e^{j \theta_{u} i}\right\} & (r-1 / 2) 2 k n+1 \leq u \leq r 2 k n\end{cases}
$$

for $r=1, \ldots, K$. In other words, we would like to solve

$$
\min _{\epsilon_{i}^{2}=1, i=1, \ldots, n}\|A \epsilon\|_{\infty}=\min _{\epsilon_{i}^{2}=1, i=1, \ldots, n}\left\|\sum_{i=1}^{n} \mathbf{a}_{i} \epsilon_{i}\right\|_{\infty}
$$

where $A$ is now a $2 k K n \times n$ real matrix. Therefore, the results of Section 3 can be readily applied to this problem. 


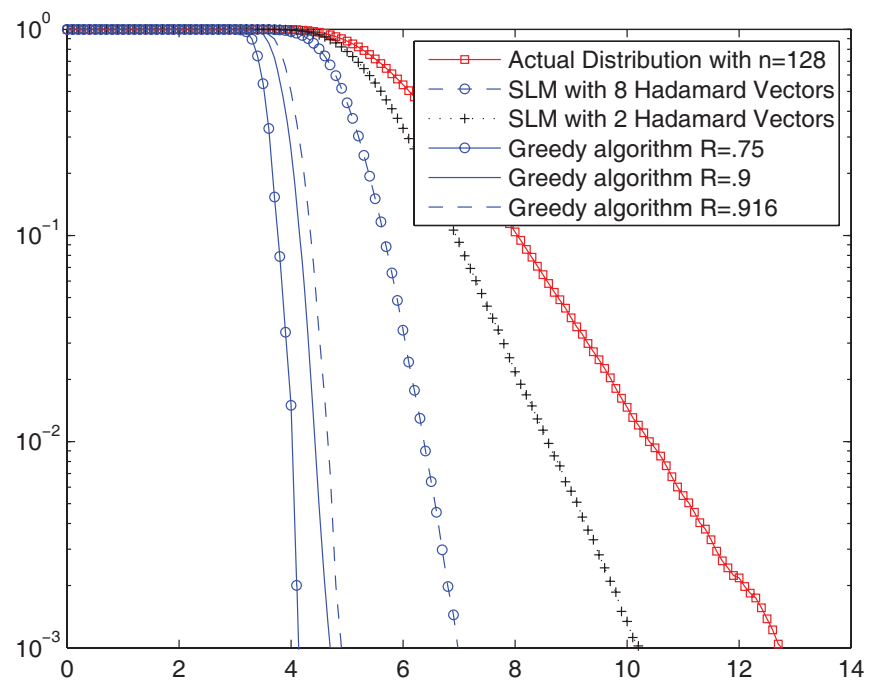

Fig. 5. PMEPR distribution for $n=128$ and using a QPSK constellation with $K=2,4$ and 6 using pruning with $m=4$ compared to the PMEPR distribution using SLM algorithm randomized by 4 and 8 Hadamard vectors.

We summarize the result in the following Corollary which is a generalization of Corollary 1. The result follows from Theorem 1 and following along the same line as in the proof of Corollary 1.

Corollary 2. Given that $c_{i}$ 's are chosen from a constellation of size q, maximum energy $P_{\max }$, and average energy $P_{a v}$, we can construct a code using the Greedy algorithm with $p=$ $\log (2 k K n)$ that has a rate $R=1-\frac{1}{K} \log _{q} 2$ and guarantees a PMEPR of $c \log (2 k K n)$ for any $n$ and $K$ where $c$ is equal to $\frac{e^{2} P_{\max }}{P_{a v} \cos ^{2} \frac{\pi}{2 k}}$ and is independent of $n$.

Remark: For large number of subcarriers, Corollary 2 implies that by letting $K=n^{\gamma}$ for any $\gamma \geq 1$, we can construct a code with rate $R=1-O\left(\frac{1}{n^{\gamma}}\right)$ and PMEPR of $c(\gamma+1) \log n$.

Fig. 5 shows the PMEPR distribution for different values of $K$ using greedy algorithm with pruning of $m=4$. This figure further compares the performance of SLM algorithm with the proposed method. Results for SLM algorithm are obtained using 4 and 8 Hadamard vectors to randomize and reduce the PMEPR of a codeword. Fig. 6 also shows the tradeoff between rate and PMEPR of a code constructing as in Corollary 2 for QPSK and 16 QAM constellations for $K=1, \ldots, 5$. Here we ignore peak values with probability less than $10^{-3}$, therefore, PMEPR is the value $\eta$ such that $\operatorname{Pr}(\operatorname{PMEPR}>\eta)=10^{-3}$. Simulation results show a significant improvement on the rate can be obtained at the price of negligible PMEPR increase. It is clear that with only $1 \mathrm{db}$ loss in the PMEPR, i.e., $10 \log (5 / 4)$, the rate loss can be significantly decreased and reduced to less than $\% 10$.

\section{CONCLUSion}

We considered the problem of finding efficiently a sign vector to minimize the PMEPR of multicarrier signals. Solving this problem requires a search over $2^{n}$ possible sign vectors. In this paper, we proposed a greedy algorithm based on $p$ norm minimization and we showed that if $p$ is properly chosen, this scheme can achieve a PMEPR of $c \log n$ with a coding rate of

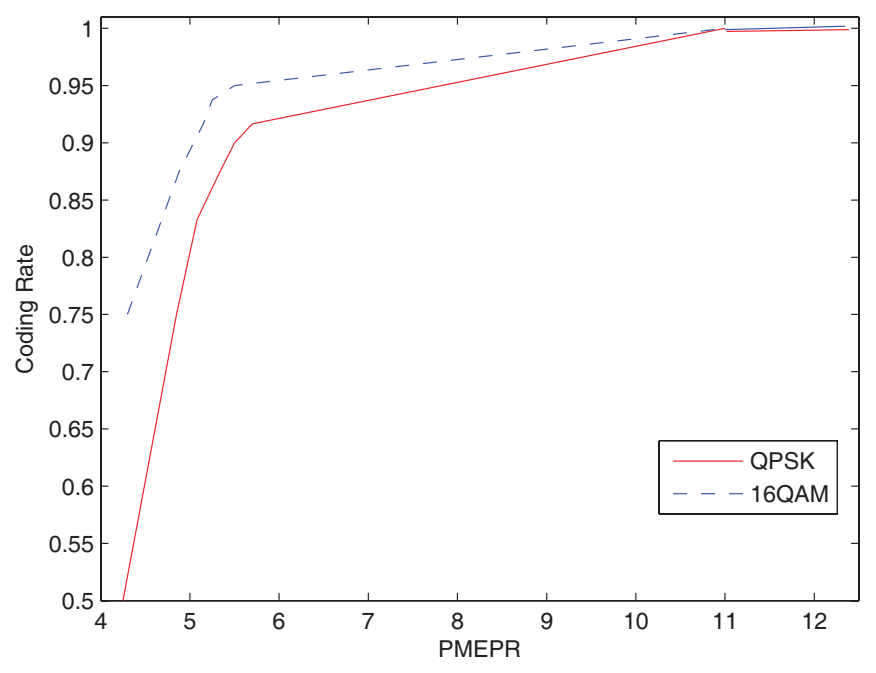

Fig. 6. Rate and PMEPR tradeoff for $n=128$ using Greedy Algorithm for different block length $K$. Here PMEPR is the value $\eta$ such that $\operatorname{Pr}(\mathrm{PMEPR}>\eta)=10^{-3}$.

$1-\log _{q} 2$ for any $n$ when a $q$-ary constellation is used. We further decreased the PMEPR by expanding the search space and using our greedy algorithm. Simulation results show that PMEPR of a multicarrier signal with 128 subcarriers can be reduced to within $1.6 \mathrm{~dB}$ of the PMEPR of a single carrier system.

In the second part of the paper, we proposed a multisymbol encoding scheme to increase the rate of the code. In this scheme, one sign vector is chosen to simultaneously minimize the PMEPR of multiple codewords. This would lead to construction of codes with rate $1-\frac{1}{K} \log _{q} 2$ and PMEPR of $c \log K n$ for any $n$ and $K$. Our simulation results show that PMEPR can be significantly reduced at the cost of a small rate loss and modest increase in the computational complexity. Our simulation results show that with only $1 \mathrm{db}$ increase in the resulting PMEPR, the rate loss can be substantially decreased and be reduced to less than $\% 10$.

\section{REFERENCES}

[1] M. Sharif and B. Hassibi, "On the existence of codes with constant PMEPR and realted designs," IEEE Trans. Signal Processing, vol. 49, no. 7, pp. 1691-1706, July 2004.

[2] A. R. Bahai and B. R. Saltzberg, Multicarrier Digital Communications: Theory and Applications of OFDM. Plenum Publishing Corporation, 1999.

[3] R. Van Nee, OFDM for Wireless Multimedia Communication. Boston, MA: Artech House, 2000.

[4] S. Litsyn, Peak Power Control in Multicarrier Communications. Cambridge University Press, 2006.

[5] K .G. Paterson and V. Tarokh, "On the existence and construction of good codes with low peak to average power ratios," IEEE Trans. Inform. Theory, vol. 46, no. 6, pp. 1974-1986, Sept. 2000.

[6] A. Mobasher and A. K. Khandani, "Integer based constellation shaping method for PAPR reduction in ofdm systems," IEEE Trans. Commun., vol. 54, no. 1, pp. 119-127, Jan. 2006.

[7] H. Minn, C. Tellambura, and V. K. Bhargava, "On the peak factor of sampled and continuous signals," IEEE Commun. Lett., vol. 5, no. 4, pp. 129-131, Apr. 2001.

[8] B. S. Krongold and D. L. Jones, "PAR reduction in OFDM via active constellation extension," IEEE Trans. Broadcasting, vol. 49, no. 3, pp. 258-268, Sept. 2003.

[9] N. Carson and T. A. Gulliver, "Peak-to-average power ratio reduction of OFDM using repeat-accumulate codes and selective mapping," in Proc. IEEE International Symp. Inform. Theory, 2002, p. 244. 
[10] H. Ochiai and H. Imai, "On the distribution of the peak to average power ratio in OFDM signals," IEEE Trans. Commun., vol. 49, no. 2, pp. 282-289, Feb. 2001.

[11] X. Li and L. J. Cimini, "Effects of clipping and filtering on the performance of OFDM," in Proc. IEEE Veh. Technol. Conf., May 1997, pp. 1634-1638.

[12] S. H. Muller and J. B. Huber, "A comparison of peak power reduction schemes for OFDM," in Proc. IEEE Global Commun. Conf., 1997, pp. $1-5$.

[13] J. Tellado and J. M. Cioffi, "Efficient algorithms for reducing PAR in multicarrier systems," in Proc. IEEE Inter. Symp. Inform., Aug. 1998, p. 191.

[14] R. Motowani and P. Raghavan, Randomized Algorithms. Cambridge University Press, 1995.

[15] S. Litsyn and A. Shpunt, "A balancing method for peak power reduction in OFDM signals," IEEE Trans. Commun., vol. 55, no. 4, pp. 683-691, Apr. 2007.

[16] M. Sharif and B. Hassibi, "High rate codes with bounded PMEPR for BPSK and other symmetric constellations," IEEE Trans. Commun., vol. 54, no. 7, pp. 71-78, July 2006.

[17] H. K. Kwok and D. L. Jones, "PAR reduction for hadamard transformbased OFDM," in Proc. 34th Conf. Signal, Systems, Computers, Princeton, NJ, Mar. 2000.

[18] L. J. Cimini and N. R. Sollenberger, "Peak to average power ratio reduction of OFDM signals using partial transmit sequences," IEEE Commun. Lett., vol. 4, no. 4, pp. 86-88, Mar. 2000.

[19] M. Breiling, S. H. Muller-Weinfurtner, and J. B. Hubber, "SLM peakpower reduction without explicit side information," IEEE Commun. Lett., vol. 4, no. 4, pp. 86-88, Mar. 2000.

[20] A. Alavi, C. Tellambura, and I. Fair, "PAPR reduction of OFDM signals using partial transmit sequence: An optimal approach using sphere decoding," IEEE Commun. Lett., vol. 9, no. 11, pp. 982-984, Nov. 2005.

[21] H. Ochiai, "A novel trellis-shaping design with both peak and average power reduction for OFDM systems," IEEE Trans. Commun., vol. 52, no. 11, pp. 1916-1926, Nov. 2004.

[22] A. Gersho, B. Gopinath, and A. M. Odlyzko, "Coefficient inaccuracy in transversal filtering," Bell Systems Tech. J., vol. 58, no. 10, pp. 23012316, Dec. 1979.

[23] G. Halasz, "On the result of Salem and Zygmund concerning random polynomials," Studia Scien. Math. Hung., pp. 369-377, 1973.

[24] M. Sharif and B. Hassibi, "On multicarrier signals where the PMEPR of a random codeword is asymptotically $\log n, "$ IEEE Trans. Inform. Theory, vol. 50, pp. 895-903, May 2004

[25] S. Litsyn and G. Wunder, "Generalized bounds on the crest factor distribution of OFDM signals with application to code design," IEEE Trans. Inform. Theory, vol. 52, no. 3, pp. 992-1006, Mar. 2006.

[26] J. Spencer, Ten lectures on the probabilistic method, SIAM CBMS-NSF Regional Conference Series Applied Mathematics, 1993.

[27] S. Litsyn and A. Yudin, "Discrete and continuous maxima in multicarrier communications," IEEE Trans. Inform. Theory, vol. 51, no. 3, pp. 919928, Mar. 2005.

[28] G. Wunder and H. Boche, "Peak value estimation of bandlimited signals from their samples, noise enhancement, and a local characterization in the neighborhood of an extremum," IEEE Trans. Signal Processing, vol. 51, no. 3, pp. 771-780, Mar. 2003.

[29] M. Sharif, M. Gharavi-Alkhansari, and B. H. Khalaj, "On the peak to average power of OFDM signals based on oversampling," IEEE Trans. Commun., vol. 51, no. 1, pp. 71-78, Jan. 2003.

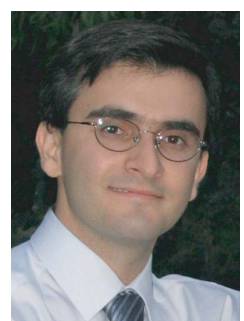

Masoud Sharif received his Ph.D. in Electrical Engineering (2005) from California Institute of Technology. In 2005, he was a post-doctoral scholar in the EE department at Caltech. Since January 2006, he has been an assistant Professor at Boston University. Dr. Sharif was awarded the C.H. Wilts Prize in 2006 for best doctoral thesis in Electrical Engineering at Caltech. He is a member of the Center for Information and Systems Engineering at Boston University. His research interests include adhoc and sensor networks, multiple-user multipleantenna communication channels, crosslayer design for wireless networks, and multi-user information theory. His recent research has focused on collaborative communication scheme in ad-hoc and sensor networks and the capacity of multiple antenna broadcast channels.

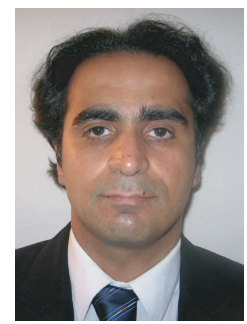

Vahid Tarokh worked at AT\&T Labs-Research and AT\&T wireless services until August 2000, where he was the head of the Department of Wireless Communications and Signal Processing. In September 2000, he joined Department of Electrical Engineering and Computer Sciences (EECS) at MIT as an associate professor. In June 2002, he joined Harvard University as a Gordon McKay Professor of Electrical Engineering . Since July 2005, he is a Hammond Vinton Hayes Senior Fellow of Electrical Engineering at Harvard University, and a Perkins professor. His research is mainly focused in the areas of Signal processing, Communications (wireline and wireless) and Networking. He has received a number of awards and holds 2 honourary degrees.

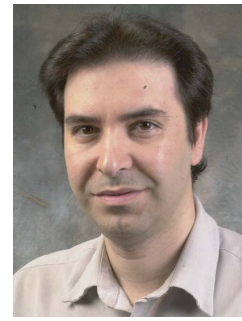

Babak Hassibi was born in Tehran, Iran, in 1967. He received the B.S. degree from the University of Tehran in 1989, and the M.S. and Ph.D. degrees from Stanford University in 1993 and 1996, respectively, all in electrical engineering. From October 1996 to October 1998 he was a research associate at the Information Systems Laboratory, Stanford University, and from November 1998 to December 2000 he was a Member of the Technical Staff in the Mathematical Sciences Research Center at Bell Laboratories, Murray Hill, NJ. Since January 2001 he has been with the department of electrical engineering at the California Institute of Technology, Pasadena, CA., where he is currently an associate professor. He has also held short-tem appointments at Ricoh California Research Center, the Indian Institute of Science, and Linkoping University, Sweden. His research interests include wireless communications, robust estimation and control, adaptive signal processing and linear algebra. He is the coauthor of the books Indefinite Quadratic Estimation and Control: A Unified Approach to H2 and H1 Theories (New York: SIAM, 1999) and Linear Estimation (Englewood Cliffs, NJ: Prentice Hall, 2000). He is a recipient of an Alborz Foundation Fellowship, the 1999 O. Hugo Schuck best paper award of the American Automatic Control Council, the 2002 National Science Foundation Career Award, the 2002 Okawa Foundation Research Grant for Information and Telecommunications, the 2003 David and Lucille Packard Fellowship for Science and Engineering and the 2003 Presidential Early Career Award for Scientists and Engineers (PECASE). He was a participant in the 2004 National Academy of Engineering "Frontiers in Engineering" program. He has been a Guest Editor for the IEEE TRANSACTIONS ON INFORMATION THEORY special issue on "space-time transmission, reception, coding and signal processing" was an Associate Editor for Communications of the IEEE TRANSACTIONS ON INFORMATION THEORY during 2004-2006, and is currently an Editor for the JOURNAL FOUNDATIONS AND TRENDS IN INFORMATION AND COMMUNICATION. 